\title{
The Effect of Aerobic Training with Purslane (Portulaca Oleracea) Seed on Toll Like Receptors in Colon Tumor Tissue of Adult Rats with Colon Cancer
}

\author{
Abdol Kheder Keshtvarz ${ }^{1}$, Maghsoud Peeri ${ }^{1 *}$, Mohammad Ali Azarbayjani ${ }^{1}$, Seyed Ali Hosseini ${ }^{2}$ \\ 1. Department of Sport Physiology, Central Tehran Branch, Islamic Azad University, Tehran, Iran \\ 2. Department of Sport Physiology, Marvdasht Branch, Islamic Azad University, Marvdasht, Iran
}

\section{Article Type: \\ Original Article \\ Article History: \\ Received: 15 May 2019 \\ Revised: 11 Jul 2019 \\ Accepted: 29 Oct 2019 \\ *Correspondence: \\ Maghsoud Peeri, Department of Sport Physiology, Central Tehran Branch, Islamic Azad University, Tehran, Iran \\ m.peeri@iauctb.ac.ir}

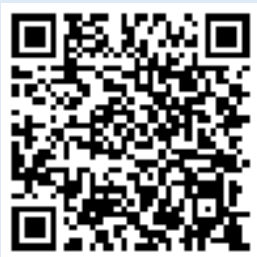

\begin{abstract}
Background and objective: Exercise and nutrition are two factors influencing the improvement of inflammatory markers in patients with colon cancer. Aim of present study was to investigate the effect of aerobic training (AT) with Purslane (Portulaca Oleracea) Seed (PS) on toll like receptor 2 (TLR-2) and TLR-4 in colon tumor tissue of rats with colon cancer.

Methods: In this experimental study 30 adults rats were divided into five groups of six rats including: 1) healthy control, 2) control, 3) training, 4) PS, and 5) training + PS. Colon cancer induced by intra-peritoneal injection of azoxymethane in groups 2- 5. During eight weeks, groups 3 and 5 performed AT for five sessions per week also groups 4 and 5 received $75 \mathrm{mg} / \mathrm{kg}$ PS intra-peritoneally. TLR2 and TLR4 protein levels were measured by ELISA method. For review the normal distribution and data Shapiro- wilk was used and for statistical analysis of data one way ANOVA with Tukey's post- hoc tests were used $(\mathrm{P} \leq 0.05)$.

Results: Training had not significant effect on TLR-2 ( $\mathrm{P}=0.91)$ and TLR-4 ( $\mathrm{P}=0.95)$; PS and training + PS significantly decreased TLR-2 and TLR-4 $(\mathrm{P}=0.001)$ also training + PS had more favorable effect on decrease of TLR-2 compare to training and PS alone $(\mathrm{P}=0.001)$.

Conclusion: Although PS alone can improve TLR-2 and TLR-4 levels in colon tumor tissue of adult rats with colon cancer, nevertheless it appears that AT along with PS have more favorable effects on improvement of TLR-2 compare to training and PS alone.
\end{abstract}

Keywords: Exercise, Portulaca, Toll-Like Receptors, Colonic Neoplasms

Copyright $@$ 2018, Jorjani Biomedicine Journal has published this work as an open access article under the terms of the Creative Commons Attribution License (http://creativecommons.org/licenses/by-nc/4.0/) which permits noncommercial uses of the work while it is properly cited. 


\section{Introduction}

One of the most common cancers of the gastrointestinal tract in young people and the elderly is intestinal cancer (1). It has been reported that in addition to genetic factors, the environmental risk factors and lifestyle have significant effects on the progress of colon cancer (2).Studies have shown that a high-fat diet, obesity, alcohol, sedentary lifestyle, lowfiber, diet, smoking, old age, diabetes, and other intestinal diseases are factors that exacerbate the negative effects of cancer (2, $3)$. These factors are likely to alter intestinal cancer through their effects on inflammatory and apoptotic factors. Apoptosis is a programmed cell death that, if not performed properly, can lead to the growth and proliferation of cancer cells. Caspase-3, Bax and Bcl-2 are important factors in regulating the apoptosis process (4). Caspase- 3 and Bax promote apoptosis, and Bcl-2 is an antiapoptotic protein $(4,5)$. Activation of toll like receptors (TLR) plays a role in the development of immune responses through inflammatory cytokines and also affects apoptosis (6). Increase in TLR-2 and TLR-4 have been shown to increase apoptosis $(6,7)$. Cancer is a serious illness, and people are advised to minimize the risk of developing the disease by increasing the uptakeof fruits and vegetables as well as reducing the consumption of fats and prepared foods. Of course, while diet and healthy eating are good ways to prevent a variety of diseases, such as cancer, the role of regular exercise should not be overlooked (8). Exercise is one of the factors that can affect inflammatory and apoptotic factors in patients with colon cancer. Various studies have been performed on the effect of exercises on inflammatory and apoptotic factors in colon cancer, and the results of studies are very contradictory (3).
Some have reported a positive effect and some a negative effect of exercises in patients with colon cancer (3). In fact, exercise is a well-known tool for dealing with chronic inflammatory conditions, but the mechanisms are not quite clear, especially when focusing on TLR signaling. Exercise can alter TLRs expression in some conditions in human and animal models (9). Although decisive reports are available on the TLR settings due to exercises, this mechanism is likely to reduce low-grade inflammation (9). Today, the use of some medicinal plant and their active ingredients has increased in improving the effects of cancer and apoptosis in cancer cells. For example, crocin has been shown to suppress apoptosis of intestinal cancer cells by suppressing nuclear factor kappa B (NF$\kappa B)(10)$. Purslane plant with scientific name of Portulaca oleracea, is a green leafy vegetable with many vitamins such as $A, B$ and $C$ (11). This plant is rich in omega-3 (11). Many studies have mentioned the role of this plant in improving inflammatory and apoptotic factors. Studies have also reported a beneficial role in reducing some of the genes associated with colon cancer (12). Due to the fact that the treatment methods used to treat colon cancer are include a combination of chemotherapy, radiation therapy and surgery, which in most cases are complex and expensive. Thus, the use of natural compounds such as medicinal plants and exercises (that have lower side effects and costs) to inhibit the tumor process and apoptosis in cancer cells is a favorable strategy to fight with cancer. Therefore, due to the limited studies on the protective effects of exercise and Purslane seed (PS) administration in the colon tissue of patients with colon cancer, the present study aimed to investigate the effect of aerobic training (AT) 
with PS on TLR-2 and TLR-4 in colon tumor tissue of adult rats with colon cancer.

\section{Materials and Methods}

In this experimental study, 30 SpragueDawley male rats (mean age: eight weeks)were purchased and in order to adapt to the new environment transferred to animal laboratory and were kept for one week under standard conditions (at a temperature of $22 \pm 2^{\circ}$ $\mathrm{C}$ and a 12-hour light/dark cycle) with free access to water and food.

\section{Groping}

The rats were divided into five groups of six rats including 1) healthy control, 2) control, 3) training, 4) PS, and 5) training + PS. In this animal model the colon cancer was induced by peritoneal injection of $10 \mathrm{mg} / \mathrm{kg}$ azoxymethane (Sigma ${ }^{\circledR}$ Product No. A2853) for 3 weeks and once per week (13). During eight weeks, groups 3 and 5 performed AT for five sessions per week (14) also groups 4 and 5 received $75 \mathrm{mg} / \mathrm{kg}$ PS (species: perpin) peritoneally (15). Forty- eight hours after the last AT session and PS administration, rats were anesthetized with ketamine and xylazine, and then the colon tumor tissue of rats was extracted and immediately frozen in nitrogen liquid. Tissue samples were stored in a freezer at -80 . C until time of measurement.

\section{Aerobic training protocol}

In order to perform AT, at first, the maximum running speed of rats was measured using the maximum running speed test on treadmill. AT sessions performed for 60 minutes of running on treadmill in three stages: warm-up, main stage and cooling down. Initially, rats ran for 5 minutes at a speed of $8 \mathrm{~m} / \mathrm{min}$ on treadmill to warm up, then ran for 50 minutes at a speed of $70 \%$ of maximum speed, and at the end ran for 5 minutes to cool down at a speed of 8 $\mathrm{m} / \mathrm{min}$.

\section{TLR-2 and TRL-4 measurement}

To homogenize, the tissues were removed from the freezer and placed in a test tube after weighing. For every $0.5 \mathrm{~g}$ of tissue, 200 Landa were added from the lysing solution and $\mathrm{A}$ protein was added to preserve the tissue proteins. After tissue homogenization using a homogenizer, the resulting solution was centrifuged for $20 \mathrm{~min}$ at $3000 \mathrm{rpm}$, and then the supernatant was carefully collected by the sampler and transferred to the microtubule. Then TLR-2 (Catalog No: DLTLR2-Ra) and TRL-4 (Catalog No: DLTLR4-Ra) protein levels were measured by ELISA method using the DEVELPO company kits.

\section{Confirmation of colon cancer}

It should be noted that the histopathology test was used to evaluate and confirm the colon cancer. Initially, vital organs were stabilized in $10 \%$ formaldehyde and passed through paraffin and implanted. The paraffin blocks were then separated to a thickness of $3 \mu \mathrm{m}$ for Hematoxylin and Eosin (H\&E) staining. The slides were examined with Olympus BX51 microscope. The abnormality degree was assessed using the following five parameters as a semi-quantitative score. A) nuclear/cytoplasm ratio (2: $25 \%<, 1: 25-50$ $\%, 0: 25 \%>$ ); B) epithelial classification (non: 0, mild: 1, severe: 2); C) nuclear curvature (non: 0, mild: 1, severe: 2); D) goblet discharge (empty to mild: 0, moderate: 1, severe 2) and E) structural disorders (non: 0, mild: 1 , severe: 2). At least five sections were reviewed for ranking. The slides were then read independently by the two evaluators without access to the files, which the 
agreement between their evaluations was more than $90 \%$. Disagreements were jointly reassessed by pathologists and a consensus decision was made. The total score of each parameter was considered as an abnormal histological score. Approximately 8 to 10 scores were calculated for all colon cancer samples, while these scores were zero to two in normal samples.

\section{Statistical analysis}

For review the normal distribution and data Shapiro- Wilk was used and for statistical analysis of data one way ANOVA with Tukey's post- hoc tests were used by GraphPad Prism software $(\mathrm{P} \leq 0.05)$. For ethical consideration, the researchers received introduction letters from Marvdasht Branch of Islamic Azad University with code IR.IAU.M.REC.1399.029.

\section{Results}

TLR-2 and TLR-4 levels are presented in Figures 1 and 2 respectively. The results of one way ANOVA test showed that there were significant differences in TLR-2 and TLR-4 levels between five study groups $(\mathrm{P}=0.001)$. The results of Tukey's post- hoc test showed that TLR-2 levels in control group were significantly higher than healthy control group $(\mathrm{P}=0.001)$ nevertheless in $\mathrm{PS}$ and training + PS groups were significantly lower than control and training groups $(\mathrm{P}=0.001)$ also in training + PS group were significantly lower than PS group $(\mathrm{P}=0.001)$ (Figure 1).

TLR-4 levels in control group were significantly higher than healthy control group $(\mathrm{P}=0.001)$ nevertheless in $\mathrm{PS}$ and training + PS groups were significantly lower than control and training groups $(\mathrm{P}=0.001)$ (Figure

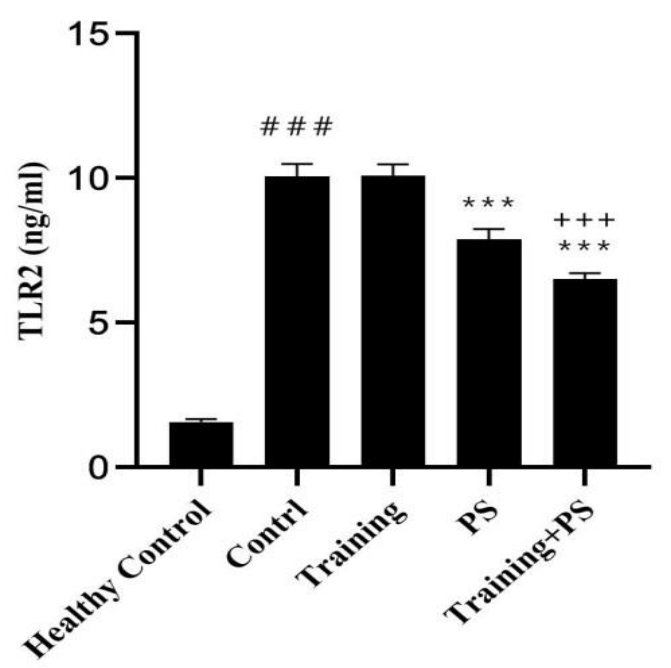

Figure 1. TLR-2 levels in five groups of study \# \# \# P $\leq 0.001$ Significant increase compare to health control group *** $\mathrm{P} \leq 0.001$ Significant decrease compare to control and training groups $+++\mathrm{P} \leq 0.001$ Significant decrease compare to PS group (PS: Purslane Seed) 


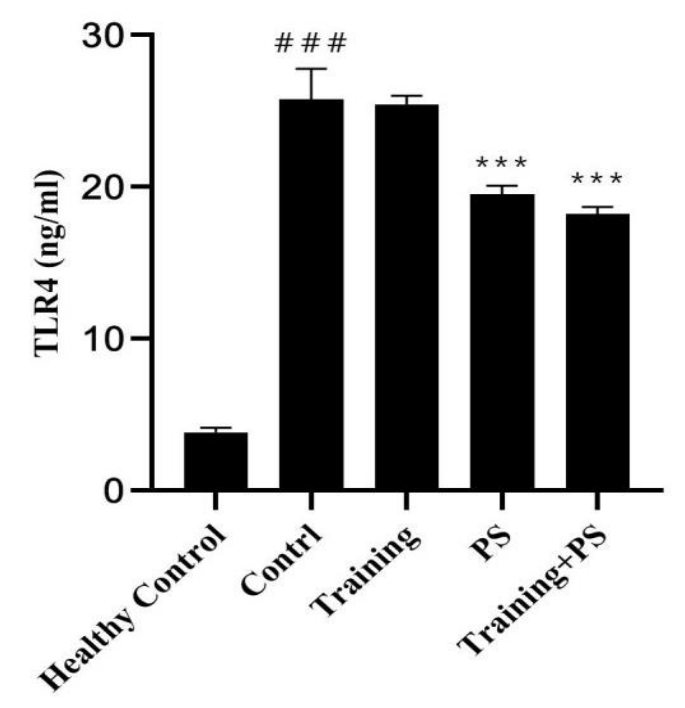

Figure 2. TLR-4 levels in five groups of study

\# \# \# $\mathrm{P} \leq 0.001$ Significant increase compare to health control group $* * * \mathrm{P} \leq 0.001$ Significant decrease compare to control and training groups

(PS: Purslane Seed)

\section{Discussion}

In present study eight weeks of aerobic training did not have a significant effect on TLR-2 and TLR-4 levels in the colon tumor tissue of rats with colon cancer.It has been shown that cancer disease and any treatment used to treat cancer (surgery, chemotherapy and radiotherapy) can cause certain side effects that affect the daily lives of patients (3).Side effects such asperipheral neuropathy, muscle weakness, change in endocrine glands, pain, cardiovascular and pulmonary complications, fatigue, immune system dysfunction, depression, anemia, skin changes, anxiety, sleep disorders, and gastrointestinal disorderscan occur during cancer and treatment (3).

Previous studies have shown that in these patients, exercise programs enhancethe quality of life along with symptoms and side effects of chemotherapy. There have been many studies on the effects of physical activity on colon cancer.The results showed that exercise through mechanisms including effects on metabolic regulation (via insulin, glucose and IGF-1), sex hormones, obesity (through adipokines such as leptin and adiponectin), oxidative stress, inflammation and immune system can play an important role in improving colon cancer $(16,17)$.In contrast with present study, it has been reported that 4 weeks of aerobic training significantly decreased TLR-4 gene expression in the lung tissue of rats (18); 6 weeks of aerobic training decreased TLR4 gene expression levels in the spinal cord tissue of rats (19) also, 6 weeks of intense interval trainings significantly decreased TLR-4 gene expression in the muscle tissue of rats (20). One of the reasons for the inconsistency of the findings of the mentioned studies with the present study could be the method of measuring the research variables (measuring protein levels in the present study versus measuring gene expression levels in the mentioned studies).Based on laboratory findings, TLR signaling appears to play a role in the anti-inflammatory effects of exercise. 
The exact physiological stimulus that mediates exercise-induced reduction in cellular TLR expression is not well known.However, some of the signals involved may be anti-inflammatory cytokines and myocytes (such as irisin, stress hormones, and heat shock proteins) secreted from the active skeletal muscle. Therefore, further research is needed to identify the exact mechanisms by which exercise or weight loss resulting from diet regulate TLR-4 expression (9).

The results showed that eight weeks of PS administration significantly decreased TLR-2 and TLR-4 levels in colon tumor tissue of rats with colon cancer. Various studies on the use of PS in the prevention and treatment of cancer have been conducted. Purslane plant is rich in omega-3 fatty acids and also contains many vitamins such as A, B and C (11). Previous studies have shown that this plant affects inflammatory and apoptotic factors (12). Some studies reported a beneficial role of PS in reducing some of the genes associated with colon cancer (21). Jin et al. (2017) showed that PSimproves intestinal cancer by suppressing the Notch1 and $\beta$ catenin genes (21). Purslane is rich in phenolic compounds, polyphenols and antioxidants, which the most important antioxidants are including alpha-tocopherol, ascorbic acid and glutathione. It has been reported that Purslane (by its strong antioxidant activity) significantly reduced the malondialdehyde which is a product of lipid peroxidation and an indicator of oxidative stress. Regarding to effects of PS on the immune system, it has been reported that PS inhibits the signaling pathway of $\mathrm{NF}-\kappa \mathrm{Bdue}$ to TNF- $\alpha$ and reduces TNF- $\alpha$ - induced inflammation; also Purslane effectively reduces the expression of intracellular interleukin- 8 which is dose dependent as well as Purslane by inhibiting the intracellular production of reactive oxygen species (ROS) and $N F-\kappa B$, reduces the expression of adhesion molecules induced by TNF- $\alpha$ (22). In confirm with the results of the present study, Zhao et al. (2107) showed that Purslane consumption can improve TLR-4 (23). Also, Zhao et al. (2019) showed that consumption of $50 \mathrm{mg} / \mathrm{kg}, 100 \mathrm{mg} / \mathrm{kg}$ and 200 $\mathrm{mg} / \mathrm{kg}$ Purslane improved TLR-4 (24). The anti-inflammatory effects of Purslane appear to be due to its antioxidant effects also Purslane is a good source of coenzyme Q10as well as contains coumarin and anthraquinone glycosides.

Regarding to interactive effects, in present study eight weeks of aerobic training along with PS significantly decreased TLR-2 and TLR-4 levels in the colon tumor tissue of rats with colon cancer, also training along with PS had favorable effect on decreasing TLR-2 and TLR-4 compare to training and had favorable effect on decreasing TLR-4 compare to PS. Therefore, it seems that PS consumption can improve the anti-inflammatory effects of aerobic training in the case of colon cancer. According to reported studies, exercise can improve the immune system through its antioxidant effects; also PS consumption through antioxidant compounds can improve the immune system. Thus it seems that aerobic training and PS through separate mechanisms lead to an improvement in the immune system in the case of colon cancer, although further studies are needed to understand the anti-inflammatory interactive effects of aerobic training and PS consumption. Limitations of the present study were included the inability to measure the TLR-2 and TLR-4 gene expression levels by Real-time PCR method, as well as the measurement of other inflammatory factors such as CRP, IL- 6 and TNF- $\alpha$. Therefore, in 
future studies, it is recommended to investigate the effects of aerobic training with different intensities along with consumption of PS with different doses on the gene expression levels of inflammatory markers in colon tumor tissue.

\section{Conclusion}

Although PS alone can improve TLR-2 and TLR-4 levels in colon tumor tissue of adult rats with colon cancer, nevertheless it appears that aerobic training along with PS have more favorable effects on improvement of TLR-2 compare to training and PS alone. Therefore, aerobic training simultaneously with PS can be used to reduce the side effects of chemotherapy in patients with colon cancer.

\section{References}

1. Bhandari A, Woodhouse M, Gupta S. Colorectal cancer is a leading cause of cancer incidence and mortality among adults younger than 50 years in the USA: a SEER-based analysis with comparison to other young-onset cancers. J Investig Med. 2017; $65 \quad$ (2): 311-5. [DOI:10.1136/jim-2016-000229]

2. Cho YA, Lee J, Oh JH, Chang HJ, Sohn DK, Shin A, et al. Genetic risk score, combined lifestyle factors and risk of colorectal cancer. Cancer Res Treat. 2019; 51 (3): 1033-40. [DOI:10.4143/crt.2018.447]

3. Oruc Z, Kaplan MA. Effect of exercise on colorectal cancer prevention and treatment. World J Gastrointest Oncol. 2019; 11 (5): 348-66. [DOI:10.4251/wjgo.v11.i5.348]

4. Jin SJ, Yang Y, Ma L, Ma BH, Ren LP, Guo $\mathrm{LC}$, et al. In vivo and in vitro induction of the apoptotic effects of oxysophoridine on colorectal cancer cells via the Bcl-2/Bax/caspase-3 signaling pathway. Oncol Lett. 2017; 14 (6): 8000-6. [DOI:10.3892/ol.2017.7227]
5. Saunders IT, Mir H, Kapur N, Singh S. Emodin inhibits colon cancer by altering BCL-2 family proteins and cell survival pathways. Cancer Cell Int. 2019; 19: 98. [DOI:10.1186/s12935-0190820-3]

6. Messaritakis I, Stogiannitsi M, Koulouridi A, Sfakianaki M, Voutsina A, Sotiriou A, et al. Evaluation of the detection of Toll-like receptors (TLRs) in cancer development and progression in patients with colorectal cancer. PLoS One. 2018; 13

(6):

e0197327.

\section{[DOI:10.1371/journal.pone.0197327]}

7. Moradi-Marjaneh R, Hassanian SM, Fiuji H, Soleimanpour S, Ferns GA, Avan A, et al. Toll like receptor signaling pathway as a potential therapeutic target in colorectal cancer. J Cell Physiol. 2018; 233 (8): 5613-22. [DOI:10.1002/jcp.26273]

8. Safari Zanjani F, Gaeini A, Kordi M, Choobineh S, Eghdam Zamiri R. Effect of concurrent training on strength \& aerobic capacity of colorectal cancer survivors. JAPD. 2017; 10 (3): 13-22.

9. Shirvani H, Soleimani M, Sanayinasab H, Rahmati-Ahmadabad S. A review on the effect of exercise on obesity by modulating the immune system and Toll- like receptors. J Mil Med. 2018; 20 (5) :456-475

10. Zhang XA, Zhang S, Yin Q, Zhang J. Quercetin induces human colon cancer cells apoptosis by inhibiting the nuclear factor-kappa B pathway. Pharmacogn Mag. 2015; 11 (42): 404-9. [DOI:10.4103/0973-1296.153096]

11. Gallo M, Conte E, Naviglio D. Analysis and comparison of the antioxidant component of Portulaca Oleracea leaves obtained by different solid- liquid extraction techniques. Antioxidants (Basel). 2017; 6: 3. [DOI:10.3390/antiox6030064]

12. Rahimi VB, Ajam F, Rakhshandeh H, Askari VR. A pharmacological review on Portulaca oleracea L.: focusing on anti-inflammatory, antioxidant, immuno- modulatory and antitumor 
activities. J Pharmacopuncture. 2019; 22 (1): 715.

13. Hajrezaie $\mathrm{M}$, Hassandarvish $\mathrm{P}$, Moghadamtousi SZ, Gwaram NS, Golbabapour S, et al. Chemopreventive evaluation of a schiff base derived copper (II) complex against azoxymethane- induced colorectal cancer in rats. 2014; PLoS ONE. 9 (3): e91246. [DOI:10.1371/journal.pone.0091246]

14. Dupas J, Feray A, Guernec A. Effect of personalized moderate exercise training on Wistar rats fed with a fructose enriched water. Nutr Metab (Lond). 2018; 15: 69. [DOI:10.1186/s12986-018-0307-6]

15. Dehghan F, Soori R, Gholami K, Abolmaesoomi M, Yusof A, Muniandy S. Purslane (Portulaca oleracea) seed consumption and aerobic training improves biomarkers associated with atherosclerosis in women with type 2 diabetes (T2D). Sci Rep. 2016; 6: 37819. [DOI:10.1038/srep37819]

16. Friedenreich CM, Shaw E, Neilson HK, Brenner DR. Epidemiology and biology of physical activity and cancer recurrence. J Mol Med (Berl). 2017; 95 (10): 1029-41. [DOI:10.1007/s00109-017-1558-9]

17. Na HK, Oliynyk S. Effects of physical activity on cancer prevention. Ann N Y Acad Sci. 2011; 176: $1229-\quad 83 . \quad[$ DOI:10.1111/j.17496632.2011.06105.x]

18. Fashi M, Agha-Alinejad H, Asilian Mahabadi H, Rezaee Seraji B, Pak Rad B. The effect of aerobic exercise in carbon black particulates air pollution on TLR4 and TNF- $\alpha$ gene expression in lung tissue of male rats. J Sport Biosci. 2015; 7 (4): 605-618.
19. Kaki A, Nikbakht M, Fathimoghadam H, Habibi A. The effect aerobic exercise on the level of expression of Toll-like receptor 4 and inflammatory mediators in the sensory spinal cord of Male Rats with diabetic neuropathic pain. Jundishapur Scie Med J. 2019; 17 (5): 503-517.

20. Sofalmanesh S, Khaledi N, Askari H. The effect of high intensity interval training on activated transcription factor 3 and Toll-like receptor 4 myocardia gene expression in diabetic rats. Sport Physiol. 2019; 11 (43): 39-54.

21. Jin H, Chen L, Wang S, Chao D. Portulaca oleracea extract can inhibitnodule formation of colon cancer stem cells by regulating gene expression of the Notch signal transduction pathway. Tumour Biol. 2017; 39 (7): 1010428317708699.

\section{[DOI:10.1177/1010428317708699]}

22. Lee AS, Kim JS, Lee YJ, Kang DG, Lee HS. Anti-TNF-alpha activity of Portulaca oleracea in vascular endothelial cells. Int J Mol Sci. 2012; 13 (5): 5628-44. [DOI:10.3390/ijms13055628]

23. Zhao R, Zhang T, Ma B, Li X. Antitumor activity of Portulaca oleracea L. polysaccharide on HeLa cells through inducing TLR4/NF- $\mathrm{BB}$ signaling. Nutr Cancer. 2017; 69 (1): 131-139. [DOI:10.1080/01635581.2017.1248294]

\begin{tabular}{|c|c|}
\hline $\begin{array}{l}\text { Zhao R, Shao X, Jia G. } \\
\text { rcinoma effect of Portulaca } \\
\text { lysaccharides by oral admi } \\
\text { testinal dendritic cells. BMC }\end{array}$ & $\begin{array}{l}\text { Anti-cervical } \\
\text { oleracea L. } \\
\text { istration on } \\
\text { Complement }\end{array}$ \\
\hline $\begin{array}{l}\text { tern } \quad \text { Med. } \quad 2019 ; \quad 19 \\
\text { OI:10.1186/s12906-019-2582-9] }\end{array}$ & (1): \\
\hline
\end{tabular}

\section{How to cite:}

Keshtvarz A.KH, Peeri M, Azarbayjani M.A, Hosseini S.A. The Effect of Aerobic Training with Purslane (Portulaca Oleracea) Seed on Toll Like Receptors in Colon Tumor Tissue of Adult Rats with Colon Cancer. Jorjani Biomedicine Journal. 2019; 7(4): 48-55. 